\title{
Ensino e pesquisa como prática profissional: entre Ciência Política, Relações Internacionais e Estudos de Defesa
}

\section{Teaching and Research as a Professional Practice: Between Political Science, International Relations and Defense Studies}

\section{MARCO CEPIK}

Em fevereiro de 2018, fui promovido para a classe de Professor Titular na Universidade Federal do Rio Grande do Sul (UFRGS). Este ensaio baseia-se no Memorial aprovado pela banca avaliadora. ${ }^{1}$

A propósito da distinção aristotélica entre memória e reminiscência (Parva Naturalia), Jacques Le Goff (1984) observou há mais de trinta anos que na Era Digital a memória individual dependeria cada vez mais de utensílios materiais e imateriais para ser evocada Le Goff (1984). Le Goff também destacou o caráter social das vivências que conferem sentido para a reminiscência. Ao mesmo tempo, as reminiscências dependem de complexos processos neurofisiológicos que tornam inseparáveis o esquecimento, as emoções e as decisões (Rolls 2014).

Dada a minha limitada capacidade de recuperar e disputar a memória individual e coletiva, procurei discutir neste texto dois aspectos da prática profissional desenvolvida nos últimos 25 anos. A primeira refere-se ao ensino de Teoria de Relações Internacionais, enquanto a segunda comenta os trabalhos produzidos em diferentes áreas. Eventuais apelos ao ethos, pathos e logos no relato da trajetória são delimitados por uma concepção da verdade como correspondência, sempre aproximativa e imperfeita, entre as proposições e a realidade externa ao autor (Aristotle 1984; Lynch 2001). ${ }^{2}$ Feita a declaração, falta o gesto. Passo ao texto.

Marco Cepik — Professor Titular, Departamento de Economia e Relações Internacionais da UFRGS. 


\section{SOBRE O ENSINO DE TEORIA DE RELAÇÕES INTERNACIONAIS}

Embora eu tenha podido ensinar e orientar em diferentes contextos institucionais uma variedade significativa de disciplinas e conteúdos, me permito um comentário sobre o ensino de Teoria de Relações Internacionais (TRI) (Cepik and Schneider 2010b).

Parto de duas premissas e uma suposição. A primeira premissa, no âmbito da Sociologia do Conhecimento, recusa o maximalismo do "programa forte” desenvolvido na Universidade de Edimburgo. Concordo com a proposição de que o conhecimento científico é social. Entretanto, considero que a produção, circulação e validação ou refutação do conhecimento não se reduz às condições sociais (Boudon 1996). As teorias de Relações Internacionais, neste sentido, também são socialmente situadas. Porém, é necessário conhecer e criticar as premissas, axiomas, conceitos, construtos, variáveis, hipóteses, métricas, indicadores, resultados e inferências que constituem teorias e modelos. A segunda premissa combina um grau moderado de relativismo epistemológico ("crenças são socialmente produzidas, mas isto não explica tudo”) com uma posição ontológica realista ("existe uma realidade externa, feita de processos físico-químicos incertos e irreversíveis”). Finalmente, suponho que haja um modo racional de estabelecer o domínio de validade de diferentes tipos de conhecimento (Prigogine and Stengers 1996).

A consistência entre tais assertivas é um tópico controverso (Joseph and Wight 2010). Seja como for, influenciaram minhas escolhas no ensino de TRI. Tento estimular uma postura crítica em relação a todas as teorias, porque as considero importantes para a prática de pesquisa e o exercício profissional na área de Relações Internacionais (Chernoff 2009). Como reivindicava a primeira geração da Escola de Frankfurt, uma atitude crítica em relação às teorias demanda avaliar de maneira integrada (não necessariamente simultânea) suas pretensões explicativas, normativas, práticas e autorreflexivas, de modo a verificar suas condições de possibilidade e domínios de validade (Horkheimer 2002).

Procuro não limitar o ensino de teoria à apresentação sequencial dos “ismos” convencionais na área de Relações Internacionais. Mesmo os bons manuais, com variações, giram em torno do Realismo, Liberalismo, Marxismo, Construtivismo, Pós-Modernismo e Feminismo (Pecequilo 2005; Nogueira and Messari 2005; Burchill et al. 2005; Reus-Smith and Snidal 2008; Carlsnaes, Risse, and Simmons 2013). ${ }^{3}$ Este é o modo predominante de ensinar TRI no Brasil e na América Latina.

Concordo com David Lake (2011) que o ensino baseado em "ismos" pode reforçar uma atitude mental escolástica, eventualmente apologética. 
Trata-se de um risco evitável (Nau 2011; Sil and Katzenstein 2011). Ainda assim, há dois problemas adicionais. Primeiro, conferir prioridade aos "ismos" tende a reificar "escolas de pensamento" (Waltz 2008; Liu and Zhang 2006, Acharya and Buzan 2010). No caso do marxismo, por exemplo, reduzi-lo a uma "escola" constitui equívoco básico. A relevância dos programas de pesquisa marxistas decorre justamente de sua diversidade e capacidade de engajar polemicamente novos problemas e fatos. Basta notar o diálogo entre marxismo político e realismo estrutural (Teschke 2003; Rosenberg 2013; Callinicos 2009). O segundo problema é a tendência a omitir do ensino as teorias de alcance médio, importantes em uma área interdisciplinar como RI (Merton 1968; Taylor 2012). ${ }^{4}$

Uma alternativa é estimular a leitura direta dos autores clássicos e contemporâneos (Mingst and Snyder 2008). Mas isto é insuficiente. É preciso analisar o desenvolvimento teórico na área em termos macroscópicos, inclusive para se evitar demasiados testes de hipóteses sem acúmulo teórico (Mearsheimer and Walt 2013).

Para lograr tal objetivo, prefiro basear a reconstrução historiográfica e teórica em algo menos genérico do que a noção de "grandes debates" (Kavalski 2007). Ou mesmo na noção de "mudança de paradigma” (Kuhn 1990). Aliás, há duas dificuldades na abordagem kuhniana. A primeira é quanto ao uso do termo paradigma. Afinal, a área de RI e mesmo as Ciências Sociais podem ser caracterizadas pela ausência de constelações de crenças amplamente compartilhadas (pré-paradigmática), ainda sem um amplo estoque de modelos e exemplos para a solução "normal” de problemas. A segunda dificuldade é que, no limite, embora Kuhn tenha rejeitado a acusação de ser radicalmente relativista, a mudança científica revolucionária para ele equivale a uma mudança de perspectiva (Gestalt) coletiva, fundada na persuasão e conversão da comunidade, sem relação necessária com os resultados cumulativos e corroborados das pesquisas. Seguir por aqui levaria ao programa forte da Sociologia do Conhecimento que recusei como ponto de partida.

Como alternativa ao critério de falseabilidade popperiano e ao critério de conversão kuhniano, decidi organizar o ensino de TRI por meio da metodologia de avaliação de programas científicos de pesquisa (MSRP). Proposta por Imre Lakatos $(1978 ; 1987)$, a MSRP tem problemas, mas é mais promissora como forma de avaliar racionalmente o nível intermediário (entre as hipóteses e paradigmas) que caracteriza o esforço de teorização (Larvor 1998). De todo modo, não é trivial definir regras explícitas, precisas e consensuais para a refutação de teorias. Tento seguir o alerta de Andrew Bennett (2003) para sermos cuidadosos em discussões metateóricas. 
Devido ao meu interesse de pesquisa em Segurança Internacional, mais espaço foi dedicado no ensino de TRI para o estudo de alguns programas de pesquisa em detrimento de outros. Mesmo sem excluir, por exemplo, os programas institucionalista ou feminista, de fato priorizei o neorrealismo estrutural, o construtivismo à Buzan, o realismo neoclássico, a Sociologia Histórica, a transição de poder, os ciclos sistêmicos de acumulação e o marxismo político.

Aos poucos, porém, as demandas práticas dos estudantes me levaram a enfatizar a discussão crítica sobre os procedimentos de formulação de teorias e modelos em Ciências Sociais. A maioria dos livros com orientação metodológica e instrumental foca, compreensivelmente, na formulação e teste de hipóteses. Ou nos diferentes delineamentos de pesquisas (Pennings, Kemanand Kleinnijenhuis 2003; Sprinz and Wolinsky-Nahmias 2004). Mas a compreensão do processo de construção de teorias explicativas e modelos descritivos precede e apoia o trabalho metodológico (Jaccard and Jacoby 2009). De modo geral, a prática de sala de aula e as interações com os orientandos me permitiram mediar a reflexão teórica e a pesquisa.

A propósito dos trabalhos publicados, defini quatro agrupamentos temáticos: Sociologia Histórica, Governança Digital, Estudos de Inteligência e Segurança Internacional. Mas as fronteiras entre os clusters são contínuas, não discretas.

Os trabalhos foram produzidos, em boa medida, reagindo a demandas e diálogos variados. Entre 1995 e 2017 , das 98 publicações principais no período (livros, artigos e capítulos de livros), 66\% foram em coautoria. Ter publicado com 42 pessoas diferentes (19 professores e 23 estudantes) provindas de áreas distintas (Ciência Política, Relações Internacionais, Engenharia de Produção, Políticas Públicas, Administração, Filosofia, Economia, Epidemiologia e Contabilidade) constituiu uma fonte importante de aprendizado, vínculo social e estímulo intelectual.

\section{SOCIOLOGIA HISTÓRICA: UM PONTO DE PARTIDA E UMA AGENDA PENDENTE}

O primeiro grupo, que chamei de Sociologia Histórica, inclui manuscritos não publicados, escritos durante a graduação e no mestrado. ${ }^{5}$ Mas o principal são três artigos publicados entre 1995 e 1999, resultados da dissertação. Voltei a publicar sobre o tema em duas ocasiões. Em 2001, para criticar o conceito de Império Mundial. E em 2017, com Pedro Brancher, para discutir a relação entre construção do Estado e evolução do sistema internacional (Cepik 1995; 1996; 1999; 2001; Cepik and Brancher 2017). 
Embora discorde de Smith (2002) quanto ao fato disso ser um problema, concordo com o seu diagnóstico de que o programa de pesquisa da Sociologia Histórica é racionalista e multicausal. ${ }^{6}$ Originado da crítica à teoria funcionalista da modernização, houve significativa dispersão na Sociologia Histórica ao longo do tempo. Mas a ancoragem principal é marxista, weberiana e braudeliana, buscando explicar macroprocessos de mudança (Skocpol 1984; Kalberg 1994; Calhoun 2003).

Meu interesse inicial pela Sociologia Histórica centrou-se em duas questões. A primeira era saber por que as revoluções sociais acontecem e, portanto, se este tipo de mudança ainda ocorreria no futuro. A segunda questão era sobre o papel do Estado e dos partidos no desenvolvimento. Ambas emergiram no contexto do debate sobre a natureza dos processos políticos que marcaram o desaparecimento do campo soviético e suas implicações (Almond 1996; Lima 1996). No começo dos anos 1990 havia forte preferência por estratégias reformistas de mudança, acudidas por um horizonte normativo que articulava o potencial emancipador do mercado e da democracia, no limite indo além do capitalismo (Reis 2000). Também fui influenciado e adotei em grande medida uma concepção reformista, mas algo continuava fora da ordem (Przeworski 1985).

Comecei por definir as revoluções modernas como uma modalidade de mudança (transformação global e violenta do sistema social) associada a um tipo de conflito (confronto estratégico intenso entre grupos humanos organizados). A mudança social está necessariamente relacionada aos conflitos, nem todos violentos, na proporção em que eles se referem a opções relevantes de futuro das sociedades (Cepik 1999). Por outro lado, os problemas organizacionais, ideológicos e políticos envolvidos na constituição de sujeitos coletivos tornam insuficiente uma abordagem puramente estrutural da mudança. Por meio de revisão da literatura e da comparação de teorias, procurei sintetizar a perspectiva estrutural de Theda Skocpol (1978) com a explicação do processo de mobilização proposta por Charles Tilly (1978; 1997; 2003). Combinando-os, é possível obter uma especificação mais precisa das condições necessárias e suficientes para o aumento das chances de que uma situação de soberania múltipla se transforme em sucesso revolucionário (Cepik 1995; 1996).

A Sociologia das Revoluções continuou se desenvolvendo como programa de pesquisa (Goldstone 1998; 2009). E os problemas de fundo continuaram existindo. O principal deles é o limite que o capitalismo impõe para a sua superação pacífica reduzindo ao invés de ampliar os espaços democráticos de controle e reforma sobre os centros de poder e riqueza não eleitos (Tilly and Tarrow 2015; Skocpol 2003). Como afirmou Barrington Moore (1983, p. 273), "os custos da moderação têm sido, pelo menos, tão 
atrozes como os da revolução, e talvez bastante superiores.” Aliás, a situação brasileira atual me fez lembrar de outra passagem: "As revoluções são raras. A reforma talvez seja ainda mais rara. E nem uma nem outra são necessárias. Os países podem ficar estagnados [...]. No mundo em modernização, quem controla o futuro é quem organiza a sua política” (Huntington 1975, 352).

Neste sentido, a Sociologia Histórica também se propôs a trazer de volta para o debate o Estado e os imperativos organizacionais na política (Evans, Rueschemeyer, and Skocpol 1985). Aqui, o estudo prévio sobre revoluções e rebeliões acautela contra a concepção do sistema internacional centrada exclusivamente no Estado e nas disputas entre elites burocráticas e empresariais (Halliday 1999). A partir dos anos 1990, dois autores impactaram a compreensão da causalidade mútua entre a expansão do capitalismo, o desenvolvimento do sistema de estados e da cidadania. Com Arrighi (1996) aprendi a tentativa de síntese entre Marx, Braudel, Schumpeter e Gramsci para explicar os ciclos de acumulação e o caos sistêmico nas transições do capitalismo histórico. Com Tilly (1985; 1996), o exame das diferenças entre as trajetórias de construção dos estados nacionais na Europa permitiu reavaliar o desafio, já presente em Robert Dahl (1997), da tolerância mútua e da própria cidadania.

No artigo de 2001, critiquei o conceito de Império Mundial, em voga até o fracasso das intervenções dos Estados Unidos no Afeganistão e Iraque. Retomei elementos de Arrighi, Bendix, Elias e Aron para argumentar que um possível governo regional multinacional, ou global, capaz de equacionar desafios de sustentabilidade fiscal, social e ambiental do desenvolvimento dependeria de maior institucionalização democrática e participação popular.

Em 2017, incorporamos de forma mais decisiva a contingência das interações conflitivas entre Estados e grupos sociais para explicar diferentes resultados estruturais no sistema internacional (Cepik and Brancher 2017; Hui 2005). Em resumo, há muito por fazer na interseção entre Sociologia Histórica, Política Comparada e História dos Sistemas Internacionais (Buzan and Little 2000; Hobson 2009). Esta foi uma agenda de pesquisa interrompida, que eu gostaria muito de voltar a desenvolver.

\section{GOVERNANÇA DIGITAL: UMA AGENDA DE PESQUISA APLICADA}

Chamei o segundo grupo de publicações de Governança Digital. ${ }^{7}$ Duas preocupações permearam os trabalhos nesta área. A primeira diz respeito ao papel da informação na expansão da cidadania e na qualificação da democracia (Webster 2006; Mossberger et al. 2008; Possamai 2016; Kneuer 
2016). A segunda, quase simétrica, é sobre o efeito das tecnologias digitais na construção da capacidade estatal, incluindo a capacidade de os governantes tomarem decisões e regularem o desenvolvimento futuro da própria tecnologia (Fountain 2001; Dunleavy et al. 2008; Margetts and Dunleavy 2013; Canabarro 2014).

No artigo sobre direito à informação, por exemplo, argumentei que era insuficiente conceber tal direito apenas como liberdade negativa, um direito civil que apenas limite a intervenção do Estado (e das empresas) na privacidade dos indivíduos (Cepik 2000, p. 50). Como se sabe, na Era Digital a privacidade encontra-se de fato sob ataque. Entretanto, o acesso e uso das informações é uma pré-condição para a participação política organizada e qualificada das pessoas. Portanto, o acesso à informação relevante depende de uma base institucional e de serviços para mediação entre Estado e sociedade civil. Neste sentido, o direito à informação envolve as três dimensões da cidadania, civil, política e social a um só tempo. Sua realização na Era Digital depende de esforços de construção estatal e de democratização mais amplos do que a defesa da privacidade (Braman 2009).

Sobre informação, cidadania e democracia na Era Digital, o grau de otimismo da literatura variou ao longo do tempo. O primeiro trabalho na área foi o livro organizado por José Eisenberg e por mim, intitulado Internet e Democracia (2002). Nossa percepção do problema se alinhava desde então com a de autores mais realistas, que enfatizaram as pré-condições, desafios e limites da e-democracia (Wilhelm 2000; Hindman 2009; Howard 2010; Sampaio 2014).

A digitalização afeta de modo distinto os países do núcleo orgânico, da semiperiferia e da periferia do sistema internacional (Rennstich 2008). Por isso analisamos as tecnologias de informação e comunicação (TICs) também do ponto de vista da Economia Política Internacional. Nossa conclusão em 2002 foi que o avanço da digitalização estressava (por meio de repetidos choques schumpeterianos de inovação desencadeados por empresas e governos do núcleo orgânico) as instituições políticas de países semiperiféricos como o Brasil. Longe de ser um fator positivo uniforme para a cidadania, a democracia e o desenvolvimento no mundo, a Era Digital tende a repor em novos patamares as desigualdades e pode, inclusive, fragilizar ainda mais a base fiscal e a legitimidade do Estado. Ao mesmo tempo, não se trata de adotar uma posição ludita contra a digitalização, mas sim de disputar o processo no âmbito internacional, nacional e local (Cepik and Eisenberg 2002, 293-314).

A partir de 2008, nova pesquisa aplicada permitiu retomar a agenda sobre Governança Digital. Priorizamos então a avaliação de e-Governo, modelos de interoperabilidade, bem como problemas de institucionalização 
da governança de TI (Cepik and Canabarro 2014). Trabalhamos com protocolos de pesquisa complementares (contraste de modelos, mapeamento de processos, análise de mercado, comparação internacional e entrevistas semiestruturadas), visando a identificar nexos causais entre desenho institucional, perfil de compras governamentais, recursos humanos e desempenho de e-governo (Cepik, Canabarro, and Possamai 2014, 11-36).

Houve avanços desde 1990, início do Sistema de Administração dos Recursos de Informação e Informática do Governo Federal (SISP). Porém, em 2010 a governança brasileira de TI era um híbrido institucional, sobrepondo modelos (burocrático, gerencialista, participativo e digital) e déficits de coordenação. O perfil das compras governamentais em TI evidenciou a concentração de empresas fornecedoras e as dificuldades na gestão de contratos, efeitos herdados e potencializados pela ideologia gerencialista dos anos 1990. Isto se refletia no baixo desempenho no e-Government Development Index (EGDI), calculado pela ONU. Ocupando a $61^{\text {a }}$ posição global, o desempenho brasileiro era ainda mais fraco em infraestrutura de telecomunicações $\left(70^{\circ}\right)$ e capital humano $\left(83^{\circ}\right)$. Isto depois de uma década de esforços do governo brasileiro.

Em suma, o empoderamento dos cidadãos e da sociedade civil prometido pelas tecnologias de informação é, na verdade, objeto de disputa política. Resolvido, ampliaria a atuação sinérgica entre o Estado e a sociedade em ambientes democráticos. Sabemos hoje, por outro lado, como o fracasso em produzir tal sinergia prejudica a capacidade estatal e oblitera a democracia (Mansell et al. 2007; Anttiroiko and Mälkiä 2007; Chadwick and Howard 2010; Pimenta and Canabarro 2014). ${ }^{8}$ Trata-se de uma agenda de pesquisa dinâmica e interdisciplinar, onde as ciências exatas e humanas podem colaborar.

\section{ESTUDOS DE INTELIGÊNCIA: DO QUASE ACASO AO ETERNO RETORNO}

O terceiro grupo é formado por trabalhos na área de Estudos de Inteligência. ${ }^{9}$ Com base na tese de doutorado, defendida em 2001 sob a orientação de Maria Regina Soares de Lima (IUPERJ-UCAM, atual IESPUERJ) e a mentoria de Domício Proença Jr. (COPPE-UFRJ), comecei a publicar os primeiros resultados já no contex to da campanha global contra o terrorismo desencadeada pelo governo Bush após os atentados de setembro de 2001. Menciono isto porque, quando iniciei a pesquisa na área, em pleno otimismo liberal da primeira metade dos anos 1990, a relevância do problema era questionada e a continuidade histórica do objeto era incerta. Desde então, a expansão dos aparatos de inteligência das grandes potên- 
cias e a crescente instabilidade estratégica entre as coalizões emergentes no mundo permitem inferir que os Estudos de Inteligência continuarão existindo como campo interdisciplinar de pesquisa (Johnson 2007; 2010; 2015; Van Puyvelde and Curtis 2016; Gill and Phythian 2016; Dover, Dylan, and Goodman 2017).

No livro Espionagem e Democracia, publicado em 2003, procurei responder a quatro perguntas: 1) O que é e como funciona a atividade de inteligência? 2) Por que surgiram e por que existem diversas agências de inteligência na maioria dos países? 3) Como o conceito de segurança nacional e o segredo governamental se articulam com as atividades de inteligência? 4) Quais os instrumentos e os limites para o controle externo sobre os serviços de inteligência em contextos democráticos?

A resposta para a primeira pergunta reconhece a natureza dual da atividade de inteligência, primariamente informacional, subsidiariamente coercitiva. Ou ao revés. Na verdade, o equilíbrio entre as duas depende dos contextos institucionais e dos níveis de conflito. A função informacional corresponde a soluções variadas, desenvolvidas por governantes e burocratas, para subsidiar processos de decisão e implementação de políticas na guerra e na paz. Sobretudo nas áreas de defesa nacional, segurança institucional, relações exteriores e segurança pública (Herman 1996). A metáfora do ciclo da atividade de inteligência (requerimento, gestão de meios, coleta, processamento, análise, disseminação, avaliação), incluindo ramificações (espionagem e especialidades técnicas de coleta), embora limitada, tem sido utilizada há décadas como heurística para analisar os problemas informacionais (Johnson 1985; Lowenthal 2015; Sabatier 2007). A função coercitiva é mais explícita nos contextos em que os serviços de inteligência operam como polícia política na repressão interna a dissidentes, mas também se manifesta regularmente no sistema internacional, nas operações de coleta (espionagem), contra-inteligência e cibersegurança, bem como no amplo leque de operações encobertas (Gill 1994; Godson 1995; Scott 2004; Poznansky 2015).

Ao longo dos anos, estimulado por orientandos e colegas, elaboramos trabalhos sobre as dinâmicas e práticas de inteligência. Um deles foi o artigo em coautoria com Christiano Ambros, sobre fatores institucionais, políticos e cognitivos que explicam as falhas de inteligência e como elas tendem a ser mais comuns na fase de análise do que na coleta de informações (Ambros and Cepik 2012). Mais recentemente, nos trabalhos em coautoria com Giovanna Kuele sobre as atividades de inteligência em Operações de Paz da ONU, voltamos a avaliar também os diferentes tipos de atuação e produtos gerados, além da evolução institucional (Cepik and Kuele 2015; 2016; Kuele and Cepik 2017). Um dos projetos de pesquisa 
que desenvolvi foi sobre técnicas estruturadas de análise de inteligência estratégica em relação a três desafios contemporâneos de segurança internacional: dissuasão nuclear, contraterrorismo e operações de paz. A controvérsia teórica envolve o realismo estrutural, a partir do qual se explica a necessidade de os países manterem sistemas de inteligência e capacidades analíticas robustas para lidar com incerteza e ganhos relativos de segurança (Phythian 2009).

A resposta para a segunda pergunta enfatiza as origens diversas e as descontinuidades históricas que caracterizam a evolução dos diferentes tipos de serviços de inteligência (Richelson 1995; Warner 2014). Os antecedentes remontam à diplomacia renascentista e moderna (no caso dos serviços de espionagem e de criptologia), à separação funcional entre inteligência e outras funções de comando no modelo prussiano de organização do estado-maior das forças armadas depois das guerras napoleônicas, bem como ao policiamento político na Europa, depois de 1848 (Cepik 2003, 85-135). Porém, só se pode falar de sistemas nacionais de inteligência institucionalizados na segunda metade do século XX. Não se trata, portanto, de um resquício absolutista ou de um mero subproduto das guerras mundiais (Zegart 1999).

Posteriormente, ampliei a pesquisa comparativa sobre sistemas de inteligência. Empiricamente, um desafio da área é ir além dos casos mais pesquisados, sobretudo Estados Unidos e Reino Unido (Davies and Gustafson 2013; Cepik 2011). Os trabalhos sobre Brasil se enquadram neste esforço (Cepik and Brandão 2003a; 2003b; Cepik 2005; 2007; Cepik and Bruneau 2008). Realizamos ainda pesquisas sobre subsistemas específicos, por exemplo, na área policial e de segurança pública (Cepik 2006; Cepik and Ambros 2013; Brandão and Cepik, 2013). O desafio é teórico e metodológico, não apenas empírico. Neste sentido, retomei estudos de caso sobre diferentes períodos da história da inteligência dos Estados Unidos, procurando reinterpretar teoricamente uma literatura que costuma ser descritiva e paroquial (Cepik 2014). Nos trabalhos comparativos com poucos casos temos testado diferentes abordagens que reputo promissoras, como a Teoria do Equilíbrio Pontuado (Cepik and Ambros 2014) e a Análise de Redes (Cepik and Möller 2017; Cepik 2017).

A terceira pergunta era sobre a compatibilidade de conceitos como segurança nacional, segredo governamental e inteligência com a democracia. A resposta simples e honesta que pude produzir foi: depende da legitimidade e da capacidade de cada Estado. Em qualquer país, a construção de sistemas de inteligência envolveu obter algum equilíbrio, tenso e precário, entre, no mínimo, os interesses dos governantes, dos governados e das burocracias especializadas (Brandão 2010). Não há solução ótima, mas 
algumas são realmente péssimas. A insegurança estrutural dos Estados nacionais no sistema internacional e os jogos aninhados (nested) que envolvem os atores relevantes tornam as soluções normativas mais comuns (tecnocráticas ou liberais) bastante problemáticas. Afinal, é “justamente para reduzir a incerteza e aumentar a capacidade de preservar a segurança nacional que existem as forças armadas, polícias e serviços de inteligência. Porém, na medida em que a própria busca de segurança é problemática, tais organizações de força e inteligência são também parte do problema” (Cepik 2003, p. 150). Ainda não voltei a este tema, mas sei que o avanço tecnológico, a privatização em grande escala e as crises econômicas e políticas vêm agravando o problema (Gill 2016).

Da mesma forma, no livro de 2003 respondi à quarta pergunta, sobre os mecanismos de controle externo, enfatizando dilemas e limites. Concluí que o controle externo sobre as instituições de segurança, defesa e inteligência poderia ser mais efetivo no âmbito de comitês parlamentares permanentes, cortes judiciais especializadas e corregedorias com pessoal especializado. Porém, como se viu na América Latina e em outros lugares, do ponto de vista da soberania popular e nacional, os próprios órgãos de controle externo e seus membros são parte do desafio democrático (Cepik 2017; Goldman and Rascoff 2016). Trata-se de uma agenda de pesquisa cada vez mais relevante na Era Digital, a qual demanda pesquisa e análise crítica.

\section{SEGURANÇA INTERNACIONAL: CONTEXTO, ESTRUTURA E INTERAÇÕES}

Finalmente, comento os trabalhos sobre Segurança Internacional. ${ }^{10}$ Ao longo dos anos, busquei uma abordagem centrada nos problemas associados ao uso da força em Segurança Internacional (Walt 1991). Por outro lado, e de modo nem sempre consistente, também fiz uso recorrente e pragmático da chamada Teoria dos Complexos Regionais de Segurança (Buzan 1991; Buzan, Wæver, and Wilde 1998; Buzan and Wæver 2003; Buzan e Hansen 2009; Buzan and Wæver 2009).

Agregarei os comentários a partir de três recortes. Inicialmente comentarei os trabalhos com foco maior em discussão conceitual e análise institucional. Em seguida, os trabalhos referidos a países, principalmente o Brasil, incluindo as questões relativas à cooperação internacional e a conexão entre meios e fins. Finalmente, comentarei os trabalhos que discutem o uso da força no sistema internacional.

Em maio de 2001, por exemplo, foi publicado o artigo Segurança Nacional e Segurança Humana: Problemas Conceituais e Consequências Políticas. Nele eu discuti a tensão entre a segurança do estado, dos indivíduos e dos diferen- 
tes grupos sociais, uma vez que não redutíveis umas às outras. Também adotei algumas definições de partida que mantive desde então: "Segurança é uma condição relativa de proteção na qual se é capaz de neutralizar ameaças discerníveis contra a existência de alguém ou de alguma coisa. Em termos organizacionais, segurança é obtida através de padrões e medidas de proteção para conjuntos definidos de informações, sistemas, instalações, comunicações, pessoal, equipamentos ou operações. As medidas de proteção devem guardar certa proporcionalidade em relação às ameaças percebidas. Na ausência de proporcionalidade, a busca de segurança torna-se, ela própria uma ameaça. $\mathrm{O}$ requisito de proporcionalidade serve para refutar a noção de segurança enquanto uma "condição absoluta de ausência de ameaça ou mesmo de incerteza” (Cepik 2001a, p. 02).

Me parece, portanto, que um uso mais restritivo e, vá lá, realista do conceito de segurança constitui medida prudencial no interesse dos setores mais fracos e oprimidos na sociedade, a serviço, portanto, da liberdade e da democracia. Pelo contrário, o uso expansivo do conceito de segurança (humana ou multidimensional) embute riscos políticos e perda de foco analítico. Logo depois da publicação do artigo problematizando os conceitos de segurança nacional e de segurança humana, nova expansão conceitual seria promovida a partir dos Estados Unidos, na esteira dos atentados terroristas de 11 de setembro de 2001. Desde então, a ameaça terrorista foi hipostasiada pela propaganda e realimentada pelo medo, demandando mais pesquisa sobre seus contornos propriamente estratégicos (Cepik 2010b; Cepik, Canabarro, and Borne 2014).

No artigo de 2012, sobre crime organizado internacional, por exemplo, procuramos realizar uma reavaliação crítica da visão, bastante disseminada, segundo a qual o parasitismo do crime organizado e as metas políticas do terrorismo e da insurgência teriam se tornado fenômenos indistintos no século XXI (Cepik and Borba 2012).

Além das razões políticas (alternativa ao neoliberalismo e o autoritarismo), a recepção favorável à Teoria dos Complexos Regionais de Segurança na América Latina decorreu de sua valorização das regiões como um nível de análise relevante, do seu esforço para combinar análise estrutural e relacional, bem como de sua modelagem relativamente simples e flexível para descrever e interpretar diferentes constelações de atores, processos, setores e níveis de análise. Por outro lado, ao tentarmos aplicar o modelo para analisar a situação contemporânea de regiões como a América do Sul e a África Austral, também foi possível identificar lacunas e inconsistências na tentativa de síntese entre o construtivismo e o realismo estrutural (Buzan, Jones, and Little 1993; ${ }^{11}$ Buzan 2010; Reeves 2014 ${ }^{12}$ ). No trabalho sobre segurança andino-brasileira, por exemplo, procuramos diversificar 
os tipos de atores (potências de outras regiões, organismos regionais e atores locais) relevantes para as dinâmicas de segurança nos Andes e na Amazônia (Cepik and Bonilla 2005). As evidências discursivas e empíricas sobre as ameaças percebidas permitiu refutar a tese dos dois subcomplexos de segurança, andino e do Cone Sul. Em trabalhos posteriores, comparei instituições de segurança regional na Europa (OSCE) e na Ásia (OCX) para avaliar a dimensão de segurança na União das Nações Sul Americanas (Cepik 2009a; 2010c). Também discuti os desafios da cooperação sul-sul em segurança e defesa (Cepik 2009b; 2010a).

Em um capítulo sobre polaridade internacional e os desafios de segurança na América Latina, procurei identificar critérios necessários e suficientes para a caracterização de um Estado como grande potência (capacidade de dissuasão nuclear crível, comando do espaço e inexpugnabilidade militar convencional). Assim, caracterizei a ordem internacional pós-Guerra Fria como sendo tripolar e desequilibrada, não unipolar. Isto tem implicações para as dinâmicas regionais de segurança e para as opções estratégicas dos demais países (Cepik 2013).

Por exemplo, no artigo de 2011 sobre a cooperação espacial chinesa no plano global e regional, compreendi que, dada a estrutura de poder tripolar no sistema internacional e a dependência crescente de todos os países em relação ao espaço, as razões chinesas para a cooperação espacial eram, simultaneamente, a busca pela segurança, pelo desenvolvimento e por legitimidade. Ao analisar o desenvolvimento do programa espacial chinês, particularmente nos campos de satélites de imagem, navegação, comunicação e retransmissão de dados, bem como nas áreas de satélites micro e nano, dados os incentivos estruturais, os objetivos estratégicos e o nível de desenvolvimento tecnológico, as iniciativas multilaterais da China junto ao Comitê das Nações Unidas para o Uso Pacífico do Espaço Exterior (CPOUS) e à Organização de Cooperação Espacial da Ásia-Pacífico (APSCO) visavam a aumentar a influência internacional de Pequim sem gerar reações exageradas dos Estados Unidos e de seus aliados, postergando uma eventual militarização do espaço.

Assim, o segundo recorte na área de segurança internacional tem como foco os problemas de segurança e defesa de alguns países, principalmente o Brasil, mas também a África do Sul e a China (Cepik 2010b; Cepik and Schneider 2010a; Cepik and Arturi 2011; Cepik 2011; Cepik and Machado 2011; Cepik and Jornada 2014; Cepik and Licks Bertol 2016).

Por exemplo, no artigo de 2016 sobre a política de defesa, procuramos verificar em que medida os objetivos estratégicos definidos pela Estratégia Nacional de Defesa (2008) estavam sendo realizados em termos de construção institucional, construção de capacidades militares, alocações orça- 
mentárias e desenvolvimento de uma base industrial de defesa. Embora moderadamente otimistas com os resultados observados até então, identificávamos naquele momento o aprofundamento da crise política e seus impactos econômicos e fiscais um risco elevado para a continuidade de projetos e para a perseverança em relação às próprias finalidades estratégicas (Cepik and Licks Bertol 2016).

Finalmente, o terceiro recorte sobre segurança internacional abarca trabalhos que analisam problemas mais diretamente relacionados ao uso da força, ainda que não diretamente o combate militar e a guerra. Por exemplo, no capítulo sobre a Defesa Nacional Antimísseis (NMD) dos Estados Unidos, além dos problemas políticos e de grande estratégia, procuramos analisar também o perfil de força (balanço nuclear entre Estados Unidos, Rússia e China) e as condições operacionais (dependentes da supressão de defesas antiaéreas e ataques em profundidade aos centros de comando e controle adversários) para a plena implementação da NMD (Cepik and Martins 2014). Em trabalhos subsequentes, discutimos desdobramentos, em termos de requisitos operacionais, de diferentes conceitos estratégicos, como o artigo sobre o conceito de defesa em camadas do Pré-Sal e o papel da América do Sul na estratégia militar brasileira (Cepik, Oliveira, and Brites 2015). E também o caso do artigo sobre o conceito de Ataque Global Imediato (Simionato and Cepik 2017).

Na Era Digital, o uso da força como um meio para atingir fins políticos tornou-se cada vez mais complexo (para usar um termo gasto) justamente pela instabilidade e disputa em torno dos fins. Mas também por causa de meios (nucleares e cibernéticos, por exemplo) que desafiam a análise racional do grau de força requerido pela escala das demandas políticas postas sobre cada lado (Diniz and Proença Júnior 2012). Por exemplo, no artigo de 2015 sobre guerra cibernética, ponderamos contra três assertivas bastante comuns. Primeiro, contra a noção de que o ciberespaço seja ou deva ser considerado um quinto domínio operacional (separado das ações em terra, ar, mar e espaço sideral). Segundo, contra a ideia de que as armas lógicas utilizadas em operações ofensivas cibernéticas causem mais dano, necessariamente, do que ataques cinéticos ou, de modo mais amplo, que as guerras convencionais tenham se tornado menos severas com o advento dos meios cibernéticos. Finalmente, disputamos o senso comum de que as capacidades ofensivas e defensivas cibernéticas de Estados, empresas e grupos não-estatais estariam se tornando equivalentes. Por extensão, a noção equivocada de que crimes cibernéticos, vulnerabilidades de segurança e guerra cibernética constituem partes convergentes de um mesmo problema que demandaria, da parte de sistemas judiciais e de defesa nacional, as mesmas leis, ferramentas e construções discursivas (Cepik, Canabarro, and Borne 2015). 
Atualmente, desenvolvo uma agenda de pesquisa focada nos mecanismos sociais que explicam os efeitos recíprocos entre mudanças contextuais (transições globais climáticas, demográficas, energéticas e tecnológicas), estruturas (polaridade e governança) e padrões de interação entre as grandes potências (polarização e cooperação) na configuração da segurança internacional.

\section{COMENTÁRIO FINAL}

No doutorado, meu professor de Política Comparada mangou que eu não seria cientista político se evitasse o núcleo duro da disciplina (sistemas partidários e comportamento eleitoral). De fato. Graduado em História, não me tornei historiador. Mestre em Ciência Política, fiz dissertação sobre Sociologia. Doutor em Ciência Política, piso territórios fronteiriços e temas ajenos. Fui professor de Política Comparada, faço pesquisa comparativa de poucos casos. Não sei muito de métodos e técnicas, mas apoio seu aprendizado. Professor de Teoria das Relações Internacionais, entendo por que meu professor de Análise Política do mestrado repudiava a figura do especialista em teoria. Procuro levar a sério os conceitos ao interpretar evidências.

Mas não sou pessoa criativa. As ideias, quando chegam, vem por laboriosas trilhas. Sou organizado, um sintoma, não uma virtude. A curiosidade e a disciplina adquiri em casa e na militância política juvenil. Gosto da profissão, dediquei parte da vida para a universidade. Tomara a família compreenda, mesmo se não puder perdoar. Deveres de fundamento a lida, empírico modo, ensina, notou o Rosa. Em resumo, cada qual com a sua loucura. Mas, como disse um outro Pessoa, sem a loucura, o que é a pessoa, mais que a besta sadia, cadáver adiado que procria?

Vim até aqui. Tive a sorte de interagir com estudantes e colegas mais capazes do que eu. Buscarei educar-me até entrar no rio Lete, a caminho do Hades. Afinal, como disse um professor de Estudos Estratégicos com quem muito aprendi sem ter sido seu aluno, educação é o que resta em nós depois que esquecemos tudo. Quero pesquisar sobre mecanismos sociais em segurança internacional e sobre a governança digital. No Brasil, na América Latina e no mundo, a década de 2020 traz desafios ordinários, extraordinários, e alguns até surreais. Como escreveu um professor de História Internacional com quem muito aprendi sem ter sido seu aluno, o descompasso entre as classes e as nações no capitalismo contemporâneo gera apartheid globalizado. Construir alternativas exige reflexões e ações coletivas com perspectiva histórica, sem ufanismos deterministas ou derrotismos mesmerizantes. 


\section{REFERÊNCIAS}

Acharya, Amitav and Barry BUZAN. 2010. Non-Western International Relations Theory: Perspectives on and Beyond Asia. London, Routledge,

Almond, Mark. 1996. Revolution: 500 years of struggle for change. London, De Agostini Editions.

Anttiroiko, Ari-Veikko, and Matti Mälkiä [Eds.]. 2007. Encyclopedia of Digital Government. London, Idea Group, Volumes I, II, and III, 1648 p.

Aristotle. 1984. "Rethoric". In The Complete Works of Aristotle, edited by Jonathan Barnes: 4.618-866. Princeton-NJ, Princeton University Press.

Arrighi, Giovanni. 1996. O Longo Século XX: Dinheiro, Poder e as Origens do nosso Tempo. São Paulo: Contraponto/Unesp.

Barros, Alejandro, Marco Cepik, and Diego Canabarro. 2010. "Para além da e-PING: o desenvolvimento de uma plataforma de interoperabilidade de e-Serviços no Brasil”. In Panorama da Interoperabilidade no Brasil, edited by Claudia Mesquita, and Nazare Bretas: 137-57. Brasília-DF, MP/SLTI.

Bennet, Andrew. 2003. "A Lakatosian Reading of Lakatos: What Can We Salvage from the Hard Core?” In Progress in International Relations Theory: Appraising the field, edited by Colin Elman, and Miriam Elman: 455-94. Cambridge-MA, BCSIA.

Boudon, Raymond. 1996. Conhecimento. In Tratado de Sociologia, edited by Raymond Boudon: 519-60. Rio de Janeiro, Jorge Zahar Editor.

Braman, Sandra. 2009. Change of State: Information, Policy, and Power. CambridgeMA, MIT Press,

Brandão, Priscila, 2010. Serviços Secretos e Democracia no Cone Sul: Premissas para uma Convivência Legítima, Eficiente e Profissional. Niterói-RJ, Impetus.

Brandao, Priscila, and Marco Cepik. [Eds.]. 2013. Inteligência de Segurança Pública: Teoria e Prática no Controle da Criminalidade. Niterói-RJ, Impetus,

Burchill, Scott et al. 2005. Theories of International Relations. New York-NY, Palgrave Macmillan, 2nd edition.

Buzan, Barry. 1991. People, States Eे Fear: An Agenda for International Security Studies in the Post-Cold War Era. Boulder-CO, Lynne Rienner. 
Buzan, Barry. 2010. China in International Society: Is 'Peaceful Rise' Possible? The Chinese Journal of International Politics 3: 5-36.

Buzan, Barry, Charles Jones, and Richard Little. 1993. The Logic of Anarchy. New York, Columbia University Press.

Buzan, Barry, and Richard Little. 2000. International Systems in world History: remaking the study of International Relations. Oxford-UK, Oxford University Press.

Buzan, Barry, and Lene Hansen. 2009 The evolution of international security studies. New York: Cambridge University Press,

Buzan, Barry, and Ole Wæver. 1998. Regions and Powers. New York, Cambridge University Press, .

"Macrosecuritisation and security constellations: reconsidering scale in securitisation theory". Review of International Studies 35: 253-76.

Buzan, Barry, Ole Wæver, and Jaap Wilde. 1998. Security: A New Framework for Analysis. Boulder-CO, Lynne Rienner,

Calhoun, Craig. 2003. "Why Historical Sociology?” In Handbook of Historical Sociology, edited by Gerard Delanty, and Engin Isin: 383-93. London, SAGE.

Callinicos, Alex. 2009. Imperialism and Global Political Economy. Cambridge, Polity Press.

Canabarro, Diego. 2014. "Governança Global da Internet: Tecnologia, Poder e Desenvolvimento". Tese Doutoral defendida junto ao PPG em Ciência Política. Porto Alegre-RS, UFRGS.

Canabarro, Diego, Ana Júlia Possamai, Marco Cepik, and Marcelo Pimenta. 2013. "Ambientes Informacionais Voltados ao Monitoramento e à Avaliação de Políticas Públicas: construindo capacidade estatal e democracia na Era Digital". XVIII Congreso Internacional sobre la Reforma del Estado y de la Administración Pública (Anales) CLAD. Montevidéu.

Carlsnaes, Walter, Thomas Risse, and Beth Simmons [Eds.]. 2013. Handbook of International Relations. Thousand Oaks-CA, Sage.

Cepik, Marco. 1995. Estrutura e Ação na Sociologia das Revoluções Modernas: Skocpol \& Tilly. Anos 90 (UFRGS), 4: 153-78. 
1996. Sociologia das Revoluções Modernas: Uma Revisão da Literatura Norte Americana. BIB Revista Brasileira de Informação Bibliográfica em Ciencias Sociais 42: 59-109,

. 1997, Informação e Decisão Governamental: Uma Contradição em Termos? IMAP. Seminário Informação, Estado e Sociedade (Anais): 27-43. Curitiba-PR.

1999. Para uma Sociologia Histórica das Revoluções Modernas. Teoria छ̊ Sociedade(UFMG) 3: 129-62.

2000, Direito à Informação: Situação Legal e Desafios. IP. Informática Pública 2, no. 2: 43-56.

2001. Do Estado Nacional ao Império Mundial? Sociedade em Debate (UCPel), 7, no. 1: 3-30.

. 2001a. Segurança Nacional e Segurança Humana: Problemas Conceituais e Consequências. Security and Defense Studies Review 1, no. 1: 1-19.

2001b. Segurança Nacional e Controle Público: Limites dos Mecanismos Institucionais Existentes. Contexto Internacional 23, no. 2: 295-359.

. 2002. Inteligência e Políticas Públicas. Security and Defense Studies Revierw 4, no. 1: 21-45,

2003. Espionagem e Democracia. Rio de Janeiro, Editora FGV,

2005. Regime político e sistema de inteligência no Brasil: legitimidade e efetividade como desafios institucionais. Dados 48, no. 1: 67-113.

2006. "Inteligência de Segurança Pública em Seis Países: Mandatos Legais e Estrutura Organizacional”. In Polícia, Democracia e Sociedade, edited by Jose Luiz Ratton Jr, and Marcelo Barros: 101-49. Rio de Janeiro-RJ, Lumen Juris.

2007. "Structural Change and Democratic Control of Intelligence in Brazil". In Reforming Intelligence: Obstacles to Democratic Control and Effectiveness, edited by Thomas Bruneau, and Steven Boraz: 149-69. Austin-TX: University of Texas Press.

2009a. "Regional Security and Integration in South America: Unasur, $\overline{\mathrm{OSCE}}$, and SCO". In The United States and Europe in a Changing World, edited by Roger Kanet, 229-51. Dordrecht, Rol.

2009b. "Segurança Nacional e Cooperação Sul-Sul: Índia, Brasil e África do Sul”. In Brasil, India e África do Sul: desafios e oportunidades para novas parcerias, 
edited by Maria Regina Soares Lima, and Mônica Hirst: 63-118. Rio de Janeiro, Paz \& Terra.

. 2010a. "Segurança Regional, Instituições de Defesa e Capacidades Militares nos Países do Fórum IBAS: Índia, África do Sul e Brasil em Perspectiva Comparada”. In G3 - Fórum de Dialogo IBAS, edited by Paulo Visentini, Marco Cepik, and Analucia Pereira, 63-149. Curitiba, Juruá.

2010b. "Combate ao Terrorismo e Estado no Brasil: avaliação critica e sugestões preliminares”. In Terrorismo e Relações Internacionais, edited by Monica Herz, and Arthur Bernardes Amaral: 121-45. Rio de Janeiro, Loyola/PUC-Rio.

.2010c. "Segurança Regional e Integração na América do Sul”. In Segurança

Internacional: práticas, conceitos, tendências, edited by Marco Cepik: 43-91. São Paulo, HUCITEC.

2011a. A política da cooperação espacial chinesa: contexto estratégico e alcance internacional. Revista de Sociologia e Política 19: 81-104.

- [Ed.]. 2011b. Inteligência Governamental: Contextos Nacionais e Desafios Contemporâneos. Niterói-RJ, Editora Impetus,

2013. "Segurança Internacional: Da Ordem Internacional aos Desafios para a América do Sul e para a Celac”. In Desafíos estratégicos del regionalismo contemporáneo: Celac e Iberoaméricaedited by Adrián Bonilla, and Isabel Álvarez: 307-24. San José, Flacso.

2014. Origens do Sistema de Inteligência dos Estados Unidos: 1775-1946. Carta Internacional 9: 3-18,

2017. Bosses and Gatekeepers: A Network Analysis of South America's Intelligence Systems. The International Journal of Intelligence and Counter Intelligence 30: 701-22,

Cepik, Marco, and Christiano Ambros. 2012. Explicando falhas de inteligência governamental: fatores histórico-institucionais, cognitivos e políticos. Varia História 28: 79-99,

2013. "Inteligência de Segurança Pública nos Estados Unidos". In Inteligência de Segurança Pública: Teoria e Prática no Controle da Criminalidade, edited by Priscila Brandão, and Marco Cepik: 21-50. Niterói-RJ, Impetus.

2014. Intelligence, Crisis, and Democracy: Institutional Punctuations in Brazil, Colombia, South Africa, and India. Intelligence and National Security 29: 523-51, 
Cepik, Marco, and Carlos Arturi. 2011. Tecnologias de informação e integração regional: desafios institucionais para a cooperação Sul-Americana na área de segurança. Dados 54: 651-92,

Cepik, Marco, Fabrício Avila, and Jose Martins. 2009. Armas estratégicas e poder no sistema internacional: o advento das armas de energia direta e seu impacto potencial sobre a guerra e a distribuição multipolar de capacidades. Contexto Internacional 31: 49-83,

Cepik, Marco, and Adrian Bonilla. 2005. Seguridad Andino-Brasileña: conceptos, actores y debates. In Agenda de Seguridad Andino Brasileña, edited by Socorro Ramirez, and Marco Cepik: 37-94. Bogotá: IEPRI-FESCOL.

Cepik, Marco, and Pedro Borba. 2012. Crime organizado e segurança internacional. Contexto Internacional 33: 375-405,

Cepik, Marco, and Pedro Brancher. 2017. Estrutura e Agência nas Relações Internacionais: Construção do Estado e Evolução do Sistema Político Internacional. Austral: Brazilian Journal of Strategy \& International Relations 6: 154-89,

Cepik, Marco, and Priscila Brandão. 2003a. Brazil’s New Intelligence System: An Institutional Assessment. The International Journal of Intelligence and Counter Intelligence 16, no. 2: 349-73.

. 2003b. "The Professionalization of Intelligence in Brazil: career path, knowledge and cultural values". In Intelligence Professionalism in the Americas, edited by Russell Swenson, and Susana Lemozy: 109-54. Washington-D.C, JMIC / NDU Press.

Cepik, Marco, and Thomas Bruneau. 2008. "Brazil: national culture of intelligence". In PSI Handbook oh Global Security and Intelligence, edited by Stuart Farson et al.: 112-29. Washington-DC, Praeger Security International.

Cepik, Marco, and Diego Canabarro. [Eds.]. 2014. Governança de TI: Transformando a Administração Pública no Brasil. Porto Alegre, Editora UFRGS.

Cepik, Marco, Diego Canabarro, and Thiago Borne. 2014. "Securitização do Ciberespaço e Terrorismo: Uma Abordagem Crítica”. In Do 11 de setembro de 2001 à guerra ao terror: reflexões sobre o terrorismo no século XXI, edited by André Souza, Reginaldo Nasser, and Rodrigo Fracalossi Moraes: 162-86. Brasília, IPEA. 2015. Cyberwar: Clausewitzian Encounters. Space \& Defense 8: 19-33.

Cepik, Marco, Diego Canabarro, Ana Júlia Possamai, Eduardo Samrsla, Andre Olivera, and Ilton Freitas. 2009. "Avaliando o Desempenho do Governo Eletrônico 
no Brasil: o Índice de Qualidade de e-Serviços Governamentais”. W3C WEB. Anais da I Conferência: 1-6. São Paulo.

Cepik, Marco, and José Eisenberg. 2002. "Internet e Instituições Políticas Semiperiféricas". In Internet e Política: Teoria e Prática da Democracia Eletrônica, edited by Marco Cepik, and José Eisenberg: 293-314. Belo Horizonte-MG: Editora da UFMG.

Cepik, Marco, José Eisenberg, and Mauro Eklund. 2001, Desenhos Institucionais em Informática Pública: Estudo Comparativo em Quatro Cidades Brasileiras. IP. Informática Pública 3, no. 2: 47-72.

Cepik, Marco, Klaus Frey, and José Eisenberg. 2002. "O Acesso à Informação". In Caminhos da Transparência, edited by Bruno Speck: 378-408. Campinas-SP: Editora Unicamp.

Cepik, Marco, and Helena Jornada. 2014. "Segurança Internacional: estrutura e risco político em mercados prioritários para o Brasil”. In Internacionalização do Brasil na Globalização, edited by André Cunha, Hélio Henkin, and Marcos Lélis: 131-74. Porto Alegre: Editora da UFRGS.

Cepik, Marco; and Giovanna Kuele. 2015. Inteligência em Operações de Paz da ONU (1945-2000). Carta Internacional 10: 21-38.

. 2016. Inteligência em Operações de Paz da ONU: Déficit Estratégico, Reformas Institucionais e Desafios Operacionais. Dados 59: 963-93.

2017. Intelligence Support to Monusco: Challenges to Peacekeeping and Security. The International Journal of Intelligence, Security, and Public Affairs 19: 44-68.

Cepik, Marco, and Frederico Licks Bertol. 2016. Defense Policy in Brazil: bridging the gap between ends and means? Defence Studies 16: 1-19.

Cepik, Marco, and Felipe Machado. 2011. O Comando do Espaço na Grande Estratégia Chinesa: Implicações para a ordem internacional contemporânea. Carta Internacional 6: 112-31.

Cepik, Marco, and Jose Martins. 2014. "Defesa Nacional Antimíssil dos EUA: lógica da preempção e implicações". In Políticas de Defesa, Inteligência e Segurança, edited by Carlos Schmidt Arturi: 14-47. Porto Alegre: Editora da UFRGS.

Cepik, Marco, and Gustavo Möller. 2017. National Intelligence Systems as Networks: Power Distribution and Organizational Risk in Brazil, Russia, India, China, and South Africa. Brazilian Political Science Review 11: 1-26. 
Cepik, Marco, Lucas Kerr Oliveira, and Pedro Brites. 2015. O pré-sal e a segurança do Atlântico Sul: a defesa em camadas e o papel da integração Sul-Americana. Revista da Escola de Guerra Naval 20: 139-64.

Cepik, Marco, and Luiza Schneider. 2010a. Defesa e Segurança na África do Sul Contemporânea. In África do Sul: História, Estado e Sociedade, edited by Paulo Visentini, and Analucia Pereira: 185-206. Brasília: Funag.

. 2010b. "Kenneth Waltz". In Os Clássicos das Relações Internacionais, edited by Marcelo Medeiros et al: 84-109. São Paulo: Hucitec.

Chadwick, Andrew, and Philip Howard [Eds.]. 2009. Routledge Handbook of Internet Politics. New York: Routledge.

Chernoff, Fred. 2009 Defending foundations for International Relations Theory. International Theory 1, no. 3: 466-77.

Dahl, Robert. 1997 [1971]. Poliarquia: Oposição e Participação. São Paulo: Edusp.

Davies, Philip, and Kristian Gustafson [Eds.]. 2013. Intelligence Elsewhere: Spies and Espionage Outside the Anglosphere. Washington-DC: Georgetown University Press.

Diniz, Eugênio, and Domício Proenca Jr. 2012. A Criterion for Settling Inconsistencies in Clausewitz's On War. Journal of Strategic Studies, DOI:10.1080 /01402390.2011.621725.

Dover, Robert, Huw Dylan, and Michael S. Goodman. 2017. The Palgrave Handbook of Security, Risk and Intelligence. New York: Palgrave Macmillan.

Dunleavy, Patrick et al. 2008. Digital Era Governance: IT corporations, the state and e-government. Oxford-UK: Oxford University Press (Revised edition).

Evans, Peter, Dietrich Rueshmeyer, and Theda Skocpol [Eds.]. 1985. Bringing the State Back In. Cambridge-UK: Cambridge University Press.

Fountain, Jane. 2001. Building the Virtual State: information technology and institutional change. Washington-DC: Brookings Institution Press.

Gill, Peter. 1994. Policing Politics: Security Intelligence and the Liberal Democratic State. London: Frank Cass.

2016. Intelligence Governance and Democratisation: a comparative analysis of the limits of reform. London: Routledge. 
Gill, Peter, and Mark Phythian. 2016. What Is Intelligence Studies? The International Journal of Intelligence, Security, and Public Affairs 18, no. 1: 5-19.

Godson, Roy. 1995. Dirty Tricks or Trump Cards: U.S. Counterintelligence and Covert Action. Washington: Brassey's.

Goldman, Zachary, and Samuel Rascoff [Eds.]. 2016. Global Intelligence Oversight. Oxford: OUP.

Goldstone, Jack [Ed.]. 1998. The Encyclopedia of Political Revolutions. WashingtonDC: Congressional Quarterly.

Goldstone, Jack. 2009. "Revolution". In The SAGE Handbook of Comparative Politics, edited by Todd Landman, and Neil Robinson: 319-47. London: SAGE.

Halliday, Fred. 1999. Revolution and World Politics: Rise and Fall of the Sixth Great Power. London: Macmillan Press.

Herman, Michael. 1996. Intelligence Power in Peace and War. Cambridge: Cambridge U Press.

Hindman, Matthew. 2009. The Myth of Digital Democracy. Princeton-NJ: Princeton University Press.

Hobson, John M. 2009. "Comparative Politics and International Relations". In The SAGE Handbook of Comparative Politics, edited by Todd Landman, and Neil Robinson: 159-74. London: SAGE.

Horkheimer, Max. 2002, Traditional and Critical Theory. In Critical Theory: selected essays, edited by Max Horkheimer 188-243. New York-NY: Continuum.

Howard, Philip. 2010. The Digital Origins of Dictatorship and Democracy: Information Technology and Political Islam. Oxford-UK: Oxford University Press.

Hui, Victoria. 2005. War and state formation in ancient China and early modern Europe. Cambridge-UK: Cambridge University Press.

Huntington, Samuel. 1975 [1968]. A Ordem Política nas Sociedades em Mudança 273, 352 e 467. Rio de Janeiro: Editora Forense-Universitária.

Jaccard, James, and Jacob Jacoby. 2009. Theory Construction and Model-Building Skills: a practical guide for Social Scientists. New York: The Guilford Press. 
Johnson, Loch. 1985. Decision Costs in the Intelligence Cycle. In Intelligence: Policy and Process, edited by A. C. Maurer, Marion Tunstall, and James Keagle: 181-98. Boulder; London: Westview Press.

Johnson, Loch [Ed.]. 2007. Handbook of Intelligence Studies. New York, Routledge, 2010. The Oxford Handbook of National Security Intelligence. Oxford-UK: Oxford University Press. 2015. Essentials of Strategic Intelligence. Washington-DC: Praeger Security International.

Joseph, Jonathan, and Colin Wight [Eds.]. 2010. Scientific Realism and International Relations. New York-NY, Palgrave Macmillan,

Kalberg, Stephen. 1994. Max Weber's Comparative-Historical Sociology. Oxford-UK, Polity Press,

Kavalski, Emilian. 2007. The fifth debate and the emergence of complex international relations theory: notes on the application of complexity theory to the study of international life. Cambridge Review of International Affairs 20, no.3 (September): 435-54.

Kneuer, Marianne. 2016. E-democracy: A new challenge for measuring democracy. International Political Science Review 37, no. 5: 666-78.

Kuele, Giovanna, and Marco Cepik. 2017. Intelligence Support to Monusco: Challenges to Peacekeeping and Security. The International Journal of Intelligence, Security, and Public Affairs 19: 44-68.

Kuhn, Thomas. 1990 [1963]. A Estrutura das Revoluções Científicas. São Paulo: Perspectiva (3. ed.).

Lakatos, Imre. 1978. The Methodology of Scientific Research Programmes. CambridgeUK: Cambridge University Press. 1987. Historia de la Ciencia y sus Reconstrucciones Racionales. Madrid: Tecnos.

Lake, David. 2011. Why "isms" Are Evil: Theory, Epistemology, and Academic Sects as Impediments to Understanding and Progress. International Studies Quarterly 55: 465-80.

Larvor, Brendan. 1998. Lakatos: An Introduction. London: Routledge. 
Le Goff, Jacques. 1984. "Memória”. In Enciclopédia Einaudi. Volume 1 (MemóriaHistória), edited by Ruggiero Romano: 11-50. Lisboa: Imprensa Nacional-Casa da Moeda.

Lima, Maria Regina Soares de. 1996. Teses Equivocadas sobre a Ordem Mundial Pós-Guerra Fria. Dados 39, no. 3.

Liu, Feng, and Ruizhuang Zhang. 2006, The Typologies of Realism. The Chinese Journal of International Politics 1: 109-35.

Lowenthal, Mark. 2015. Intelligence: From Secrets to Policy. Washington: SAGE/ CQ Press (6th edition).

Lynch, Michael P. [Ed.]. 2001. The Nature of Truth: Classic and Contemporary Perspectives. Cambridge-MA: The MIT Press. 802 p.

Mansell, Robin et al. [Eds.]. 2007. The Oxford Handbook of Information and Communication Technologies. Oxford-UK: Oxford University Press.

Margetts, Helen, and Patrick Dunleavy. 2013. The second wave of digital-era governance: a quasi-paradigm for government on the Web. Philosophical Transactions of the Royal Society, A 371: 20120382.

Mearsheimer, John, and Stephen Walt. 2013. Leaving Theory Behind: Why Hypothesis Testing Has Become Bad for IR. HKS Faculty Research Working Paper Series RWP 13-001 (January).

Merton, Robert. 1968. Social Theory and Social Structure: 39. New York: Free Press (Third expanded edition).

Mingst, Karen, and Jack Snyder [Eds.]. 2008. Essential Readings in World Politics. New York-NY: W.W. Norton \& Company (3rd Edition).

Moore, Barrington. 1983 [1967]. As Origens Sociais da Ditadura e da Democracia: senhores e camponeses na construção do mundo moderno. São Paulo: Martins Fontes (p. 497).

Mossberger, Kate, Caroline Tolbert, and Ramona McNeal. 2008. Digital citizenship: the internet, society, and participation. Cambridge-MA: MIT Press.

Nau, Henry R. 2011. No alternative to "isms". International Studies Quarterly 55: $487-91$.

Nogueira, João, and Nizar Messari. 2005. Teoria das Relações Internacionais: correntes e debates. Rio de Janeiro: Elsevier. 
Pecequilo, Cristina. 2005. Introdução às Relações Internacionais: temas, atores e visões. Petrópolis-RJ: Vozes.

Pennings, Paul, Hans Kemanand, and Jan Kleinnijenhuis. 2003. Doing Research in Political Science: an introduction to comparative methods and statistics. London: Sage.

Phythian, Mark. 2009. "Intelligence Theory and Theories of International Relations: shared world or separate world?" In Intelligence Theory: key questions and debates, edited by Peter Gill, Stephen Marrin, and Mark Phythian: 54-72. New York: Routledge.

Pimenta, Marcelo and Diego Canabarro [Eds.]. 2014. Governança Digital. Porto Alegre-RS, Editora da UFRGS.

Possamai, Ana Júlia. 2016. Dados Abertos no Governo Federal Brasileiro: desafios de transparência e interoperabilidade. Tese de Doutorado - PPG em Ciência Política. Porto Alegre-RS, UFRGS.

Poznansky, Michael. 2015. Stasis or Decay? Reconciling Covert War and Democratic Peace. International Studies Quarterly 59: 815-26.

Prigogine, Ilya, and Isabelle Stengers. 1996. The End of Certainty: Time, Chaos, and the New Laws of Nature. New York-NY: The Free Press.

Przeworski, Adam. 1991 [1985]. Capitalismo e Social-Democracia. São Paulo: Companhia das Letras.

Reeves, Jeffrey. 2014. Structural Power, the Copenhagen School and Threats to Chinese Security. The China Quarterly 217: 140-61.

Reis, Fábio W. 2000. "Para Pensar Transições: democracia, mercado, estado”. In Mercado e Utopia: teoria política e sociedade brasileira, edited by Fábio W. Reis: 16189. São Paulo: Edusp.

Rennstich, Joachim. 2008. The Making of a Digital World: The Evolution of Technological Change and How It Shaped our World. New York: Palgrave Macmillan.

Reus-Smith, Christian, and Duncan Snidal [Eds.]. 2008. Oxford Handbook of International Relations. Oxford-UK: Oxford University Press.

Richelson, Jeffrey T. 1995. A Century of Spies: Intelligence in the Twentieth Century. Oxford-UK: Oxford University Press.

Rolls, Edmund T. 2014. Emotion and Decision-Making Explained. Oxford-UK: Oxford University Press. 
Rosenberg, Justin. 2013, Kenneth Waltz and Leon Trotsky: Anarchy in the mirror of uneven and combined development. International Politics 50, no.2: 183-230.

Sabatier, Paul. 2007 [Ed.]. Theories of the Policy Process. Cambridge-MA: Westview Press (2nd edition).

Sampaio, Rafael. 2014. Orçamentos Participativos Digitais: Um mapeamento mundial das experiências já realizadas e suas contribuições para e-Participação e e-Democracia. Tese - Doutorado - PPG em Comunicação e Cultura Contemporânea. Salvador-BA: UFBA.

Scott, Len. 2004. Secret Intelligence, Covert Action and Clandestine Diplomacy. Intelligence and National Security 19, no. 2: 322-41.

Sil, Rudra, and Peter J. Katzenstein. 2011. De-Centering, Not Discarding, the "Isms": Some Friendly Amendments. International Studies Quarterly 55. no. 481-85.

Simionato, Guilherme, and Marco Cepik. 2017. O conceito de Ataque Global Imediato: premissas equivocadas, consequências perigosas. Carta Internacional 12: $5-29$.

Skocpol, Theda. 1978. "States and Social Revolutions: a comparative analysis of France, Russia, and China”. In From Mobilization to Revolution, edited by Charles Tilly. New York: Random House.

[Ed.]. 1984. Vision and Method in Historical Sociology. Cambridge-UK: Cambridge University Press.

2003. Diminished Democracy: from membership to management in american civic life. Norman-OK: Oklahoma University Press.

Skocpol, Theda. 2015. States and Social Revolutions: a comparative analysis of France, Russia, and China.

Smith, Steve. 2002. "Historical Sociology in International Relations Theory". In Historical Sociology of International Relations,m edited by Stephen Hobden, and John Hobson 223-43. Cambridge-UK: Cambridge University Press.

Sprinz, Detlef, and Yael Wolinsky-Nahmias [Eds.]. 2004. Models, Numbers छ Cases: methods for studying international relations. Ann Arbor-MI: The University of Michigan Press.

Taylor, Mark Z. 2012, Toward an International Relations Theory of National Innovation Rates. Security Studies 21, no. 1 (January): 113-52. 
Teschke, Benno. 2003. The Myth of 1648: Class, Geopolitics and the Making of Modern International Relations. London: Verso.

Tilly, Charles. 1978. From Mobilization to Revolution. New York: Random House.

War Making and State Making as Organized Crime. In Bringing the State Back In, edited by Peter Evans, Dietrich Rueshmeyer, and Theda Skocpol: 169-91. Cambridge-UK: Cambridge University Press. EDUSP.

1996 [1990]. Coerção, Capital e Estados Europeus (1990-1992). São Paulo: 1997. Does Modernization Breed Revolution? In Roads from Past to Future, edited by Charles Tilly: 109-32. Lanham-MD: Rowman \& Littlefield Publishers.

University Press.

. 2003. The Politics of Collective Violence. Cambridge-UK: Cambridge

Tilly, Charles, and Sidney Tarrow. 2015. Contentious Politics. Oxford-UK: Oxford University Press (Second fully revised edition).

Van Puyvelde, Damien, and Sean Curtis. 2016. "Standing on the shoulders of giants": diversity and scholarship in Intelligence Studies. Intelligence and National Security 31. no. 7.

Walt, Stephen M. 1991. The Renaissance of Security Studies. International Studies Quarterly 35: 211-39.

Waltz, Kenneth. 2008. "Realist Thought and Neorealist Theory". In Realism and International Politics, edited by Kenneth Waltz: 67-82. New York: Routledge.

Warner, Michael. 2014. The Rise and Fall of Intelligence: An International Security History. Washington-DC: Georgetown University Press.

Webster, Frank. 2006. Theories of the Information Society. New York: Routledge (Third edition).

Wilhelm, Anthony. 2000. Democracy in the Digital Age: challenges to political life in cyberspace. New York-NY: Routledge.

Zegart, Amy. 1999. Flawed by design: the evolution of the CIA, JCS, and NSC. Stanford-CA: Stanford University Press. 


\section{NOTAS}

1. Versão completa do Memorial em: http://professor.ufrgs.br/marcocepik/publications/memorial.

2. Sobre o conceito de verdade, ver LYNCH (2001).

3. O melhor deles, para os meus propósitos didáticos, é Carlsnaes, Risse, and Simmons (2013).

4. Teorias de alcance médio seriam aquelas que: "lie between the minor but necessary working hypotheses that evolve in abundance during day-to-day research and the all-inclusive systematic efforts to develop a unified theory that will explain all the observed uniformities of social behavior, social organization and social change" (Merton 1968). Um exemplo em RI seria Taylor (2012).

5. Seguem alguns títulos: "Brasil: movimentos insurgentes na Colónia e no Império". "Desobediência Civl e Protesto de Massas na Independência da Índia"; "Entre a reforma e a revolução: Chile 19701973"; "A Revolução de 1383 em Portugal”; "Notas sobre Rebeliões: Tilly e Rudé"; "Crise Hegemônica e Golpe Militar nos anos 1960: Brasil, Argentina e Peru”.

6. A preferência de Smith seria por uma aproximação maior entre a Sociologia Histórica e as abordagens pós-positivistas em Relações Internacionais. Mas ele próprio reconhece não ser o caso e lamenta.

7 Cepik 1997: 27-43; Cepik 2000: 43-56; Cepik, Eisenberg e Eklund 2001: 47-72; Cepik, Frey e Eisenberg 2002: 378-408; Cepik e Eisenberg 2002: 293-314; Cepik et al. 2009: 1-6; Barros, Cepik e Canabarro 2010: 137-157; Canabarro, Possamai, Cepik e Pimenta 2013. As últimas publicações nesta área ocorreram quando lançamos a nova edição do livro: Cepik e Canabarro 2014. Naquele livro, agradeço ter tido a oportunidade de escrever diversos capítulos em coautoria com Diego Canabarro, Ana Júlia Possamai e Fernando Sebben.

8. Daí também a parceria entre o CEGOV e o INCT-CEADD (Centro de Estudos Avançados em Democracia Digital), sediado na UFBA.

9. Cepik 2002; Cepik e Brandão 2003a; Cepik 2003; Cepik e Brandão 2003b; Cepik 2005; 2006; 2007; Cepik e Bruneau 2008; Cepik 2011; Cepik e Ambros 2012; Brandao e Cepik 2013; Cepik e Ambros 2013. Cepik 2014; Cepik e Ambros 2014; Cepik e Kuele 2015; 2016; Cepik e Möller 2017; Cepik e Kuele 2017; Cepik 2017).

10. Cepik 2001a; 2001b; Cepik e Bonilla 2005; Cepik 2009a; 2009b; Cepik, Avila e Martins 2009; Cepik 2010a; 2010b; Cepik e Schneider 2010a; Cepik 2010c; Cepik e Borba 2011; Cepik e Arturi 2011; Cepik 2011; Cepik e Machado 2011; Cepik 2013; Cepik e Jornada 2014; Cepik e 
Martins 2014; Cepik, Canabarro e Borne 2014; 2015; Cepik, Oliveira e Brites 2015; Cepik e Licks Bertol 2016; Simionato e Cepik 2017.

11. O trabalho de 1993 foi importante para tratar teoricamente o tema da "baixa diferenciação funcional" de maneira mais dinâmica.

12. Sobre o caso chinês: Buzan 2010; Reeves 2014. 
ENSINO E PESQUISA COMO PRÁTICA PROFISSIONAL:

ENTRE CIÊNCIA POLÍTICA, RELAÇÕES INTERNACIONAIS

E ESTUDOS DE DEFESA

\section{RESUMO}

Este tex to discute duas dimensões da prática profissional de um professor e pesquisador da área de Ciência Política, Relações Internacionais e Estudos de Defesa. A primeira parte do texto explicita fundamentos do ensino de Teoria de Relações Internacionais. As seções subsequentes discutem os conteúdos da produção científica (a maior parte em coautoria) nas subáreas de Sociologia Histórica, Governança Digital, Estudos de Inteligência e Segurança Internacional. Atualmente, pesquiso os mecanismos sociais que explicam os efeitos recíprocos entre mudanças contextuais (transições globais climáticas, demográficas, energéticas e tecnológicas), estruturas (polaridade e governança) e padrões de interação entre as grandes potências (polarização e cooperação) na configuração da segurança internacional.

Palavras-chave: Defesa; Segurança; Relações Internacionais; Digital.

\section{ABSTRACT}

This text discusses two dimensions of a professor and researcher's professional practice in the area of Political Science, International Relations, and Defense Studies. The first part of the text aims at making explicit the foundations of International Relations Theory teaching. Subsequent sections discuss the scientific production (mostly co-authored) in the sub-areas of Historical Sociology, Digital Governance, Intelligence Studies, and International Security. Currently, I research the social mechanisms that explain the reciprocal effects between contextual changes (global climatic, demographic, energy, and technological transitions), structures (polarity and governance), and patterns of interaction between the great powers (polarization and cooperation) in the international security realm.

Keywords: Defense; Security; International Relations; Digital. 